\title{
CARACTERIZACIÓN AGRONÓMICA Y MORFOLÓGICA DE MAÍCES NATIVOS DEL NOROCCIDENTE DE MÉXICO
}

\author{
AGRONOMIC AND MORPHOLOGICAL CHARACTERIZATION OF NATIVE MAIZES FROM \\ NORTH-WESTERN MÉXICO
}

\author{
José G. Martín López ${ }^{1}$, José Ron Parra ${ }^{1 *}$, José J. Sánchez González ${ }^{1}$, Lino De la Cruz Larios ${ }^{1}$, Moisés M. \\ Morales Rivera ${ }^{1}$, José A. Carrera Valtierra ${ }^{2}$, Alejandro Ortega Corona ${ }^{3}$, Víctor A. Vidal Martínez ${ }^{4}$ \\ y Manuel de J. Guerrero Herrera ${ }^{3}$
}

\begin{abstract}
${ }^{1}$ Instituto de Manejo y Aprovechamiento de Recursos Fitogenéticos (IMAREFI). Centro Universitario de Ciencias Biológicas y Agropecuarias (CUCBA), Universidad de Guadalajara, Km. 15.5 Carretera Guadalajara-Nogales. Apartado Postal 129, 45110, Las Agujas, Nextipac, Mpio de Zapopan, Jalisco, México. Tel. y Fax: (33) 368207 43. ${ }^{2}$ Centro Regional Universitario Centro Occidente. Universidad Autónoma Chapingo. 58170, Periférico Independencia Poniente No. 1000, Colonia Lomas del Valle, Morelia, Michoacán, México. ${ }^{3}$ Campo Experimental Valle del Yaqui, Instituto Nacional de Investigaciones Forestales, Agrícolas y Pecuarias (INIFAP). Calle Norman E. Borlaug Km. 12 Cd. Obregón, Son. ${ }^{4}$ Campo Experimental Santiago Ixcuintla, INIFAP. Km. 6 Carretera Guadalajara-Nogales Entronque a Santiago Ixcuintla, Nayarit, México.
\end{abstract}

*Autor para correspondencia (jron@cucba.udg.mx)

\section{RESUMEN}

En este trabajo se evaluaron y caracterizaron maíces (Zea mays L.) criollos del noroccidente de México, colectados en los Estados de Jalisco, Nayarit, Michoacán y Sinaloa. Se evaluaron 90 materiales en tres localidades de Jalisco en 2005, bajo condiciones de temporal o secano, en cuanto a rendimiento de grano y otras variables agronómicas, mediante un diseño experimental de látice rectangular $10 \times 9$ con tres repeticiones. En adición, en una localidad se caracterizaron 129 materiales mediante 36 variables morfológicas y gráficas "biplot" y dendrogramas. Las mejores colectas para rendimiento y otras características de importancia fueron M05100 (Tabloncillo Perla) y M05021 (Tabloncillo), así como Tabloncillo $\mathrm{RC}_{1}$, pero distaron mucho del grupo donde se ubicó la cruza entre razas representativas de la raza Tabloncillo (JAL43 $x$ JAL263) y la mayoría de las colectas de la raza Tabloncillo Perla. Los grupos se definieron mayormente por la altitud del sitio de recolección, y el grupo más compacto y consistente fue el de los maíces pertenecientes a la raza Mushito colectados en altitudes generalmente superiores a $2000 \mathrm{~m}$; este grupo destacó por su mayor espesor del grano y longitud de la rama principal de la espiga. Las colectas representativas de la razas Tabloncillo y Tabloncillo Perla formaron un grupo compacto que se distinguió por su precocidad y porte de planta bajo. Los maíces provenientes de generaciones avanzadas de híbridos comerciales se asociaron principalmente con colectas pertenecientes a las razas de Celaya y Tuxpeño. M05002, destinado al mercado de grano blanco para "pozole", fue el mejor al resto de la raza Ancho y a los de las razas Elotes Occidentales, Elotero de Sinaloa y Bofo, utilizados también para "pozole" y "elote".

Palabras clave: Zea mays, maíces nativos, recursos genéticos.

\section{SUMMARY}

In this study we evaluated and characterized maize (Zea mays L.) landraces collected in north-western México in the States of Jalisco, Nayarit, Michoacán and Sinaloa. A total of $\mathbf{9 0}$ materials were evaluated at three locations of Jalisco in 2005, under rainfed conditions, for grain yield and other agronomic traits, using a rectangular lattice design $10 \times 9$ with three replications. Characterization was done in one location on 129 materials by means of 36 morphological traits and by using "biplot" and dendrograms graphs. The best accessions across environments for grain yield and some additional important traits were M05100 (Tabloncillo Perla), M05021 (Tabloncillo) and Tabloncillo $\mathrm{BC}_{1}$, but they were far away of the group where the cross representative of the Tabloncillo (JAL43 x JAL263), and where most collections of Tabloncillo Perla races were located. Groups were defined mainly by altitude of the collection site; the best defined group was that of landraces classified as Mushito, collected at elevations generally higher than $2000 \mathrm{~m}$; this group was distinguished by having higher values of kernel thickness and length of the main tassel branch. Accessions of races Tabloncillo y Tabloncillo Perla formed a group that was defined by their earliness and short plant height. The collections derived from hybrids advanced generations were related to collections of races Celaya and Tuxpeño. M05002 used as white grain to prepare specialty food ("pozole") was the best to the other accession of the Ancho and others of Elotes Occidentales, Elotero de Sinaloa y Bofo, used as "corn on the cob" and "pozole".

Index words: Zea mays, native varieties, genetic resources.

\section{INTRODUCCIÓN}

En diversos foros y obras culturales, técnicas y científicas se ha reconocido y destacado la diversidad de los maíces mexicanos y su importancia en los aspectos socioeconómicos y culinarios (Esteva y Marielle, 2003; González, 2006; Muñoz, 2003). Esta riqueza genética ha jugado un papel importante en México ya que se siembran variedades nativas de maíz (Zea mays L.) en regiones, áreas y nichos ecológicos donde las variedades mejoradas no expresan su potencial de rendimiento (Muñoz, 2003) o no cumplen con los niveles de calidad requeridos por los productores en la preparación de alimentos para humanos 
y animales domésticos (Ron et al., 2006). La diversidad total de maíces nativos de México podría representarse por 59 razas (Ron et al., 2006).

En el centro-occidente de México con áreas y regiones de altitudes intermedias y bajas (0 a $1800 \mathrm{msnm}$ ) y ambientes favorables para la producción de cultivos, las variedades mejoradas han estado remplazando a los maíces nativos y éstos quedan relegados a superficies reducidas o áreas montañosas, en donde aun en condiciones desfavorables se siembran predominantemente para autoconsumo bajo la custodia principalmente de agricultores de edad avanzada.

La pérdida continua de la diversidad de los maíces nativos ha renovado el interés por su rescate, conservación y aprovechamiento, de tal forma que a partir de 2003 se han apoyado proyectos para recolectar, evaluar y caracterizar maíces nativos en diferentes regiones y estados de México con diversas fuentes de financiamiento (Sistema Nacional de Recursos Fitogenéticos, Consejo Nacional de Ciencia y Tecnología y empresas privadas). Como parte de esta estrategia, se han colectado poblaciones nativas en la región noroccidente de México, por el Instituto de Manejo y Aprovechamiento de Recursos Fitogenéticos (IMAREFI) del Centro Universitario en Ciencias Biológicas y Agropecuarias (CUCBA) de la Universidad de Guadalajara, los centros regionales de Guadalajara y Morelia (CRUCOGuadalajara y CRUCO-Morelia) de la Universidad Autónoma Chapingo (UACH), y los campos experimentales de Ciudad Obregón, Son. y Santiago Ixcuintla, Nay. del Instituto Nacional de Investigaciones Forestales, Agrícolas y Pecuarias (INIFAP).

Estas colectas recientes se conservan en bancos de germoplasma para su aprovechamiento a futuro, pero requiere caracterizarlas para su uso óptimo. Los objetivos de este estudio fueron: 1) Evaluar el rendimiento de grano y otras características de importancia agronómica en 86 maíces nativos colectados recientemente en el noroccidente de México; 2) Conocer las interrelaciones de 127 maíces nativos, incluidos los anteriores, mediante características morfológicas de planta, espiga y mazorca, principalmente; y 3) Conocer la relación de estos maíces nativos con maíces mejorados y los típicos representativos o relacionados de la raza Tabloncillo predominante en la región.

\section{MATERIALES Y MÉTODOS}

El material biológico consistió en 127 accesiones de maíz del noroccidente de México colectadas en los Estados de Michoacán, Jalisco, Nayarit y Sinaloa; de éstas, 99 fueron colectadas por el IMAREFI y CRUCO-Morelia y se conservan en el banco de germoplasma del CUCBA (Ron $e t$ $a l ., 2006)$, y 28 por el INIFAP) y que se conservan en el Campo Experimental de Ciudad Obregón, Son.

En un experimento de 90 entradas se evaluaron 86 accesiones y cuatro testigos que fueron: una cruza simple experimental mejorada de la Universidad de Guadalajara (LUG03 x LUG14), una cruza entre dos colectas típicas de la raza Tabloncillo (JAL43 x JAL263) en $F_{1}$ y $F_{2}$, y la retrocruza de Tabloncillo x V530 $\left(\mathrm{RC}_{1}\right)$ proporcionada por el Dr. Fidel Márquez Sánchez de la UACH. El experimento se sembró en un diseño de látice rectangular $10 \mathrm{x}$ 9 en parcelas de dos surcos con 40 plantas, a una densidad de 50 mil plantas por hectárea en tres localidades de Jalisco: Campo Experimental del CUCBA en Zapopan (1650 msnm), Las Garzas, Mpio. de Guachinango (800 msnm) y Campo Experimental Altos-Centro de Jalisco del INIFAP en Tepatitlán de Morelos (1900 msnm). Por falta de semilla, en Las Garzas se remplazaron tres colectas (INIFAP48, INIFAP50 e INIFAP57) por tres materiales mejorados (REMACO 38, REMACO 3A y SINTA x SINTCA) que se consideraron como testigos mejorados. Los experimentos se sembraron en condiciones de temporal o secano, con el manejo agronómico recomendado por el INIFAP en cada sitio.

Los datos registrados por parcela fueron: rendimiento (REND), floración masculina (FM), floración femenina (FF), altura de planta (PL), altura de mazorca (MZ), acame de raíz (RA), acame de tallo (TA), mazorcas dañadas (MD), mazorcas por planta (MXP), calificación de mazorca (CM), longitud de la mazorca (LMZ), diámetro de la mazorca (DMZ) y porcentaje de grano (G). En Tepatitlán no se midió altura de planta, acame de raíz, mazorcas dañadas y la calificación de mazorca. Con los datos de cada ambiente se hicieron análisis de varianza individuales por localidad y un análisis combinado con los tres ambientes.

La caracterización se hizo en las 127 accesiones, que incluyeron a las 86 en la evaluación originarias de áreas tropicales y subtropicales de Jalisco, Nayarit y Sinaloa, y 41 de partes altas de Michoacán colectadas generalmente arriba de $2000 \mathrm{msnm}$. Además se incluyeron la retrocruza de Tabloncillo $\left(\mathrm{RC}_{1}\right)$ y la cruza entre colectas representativas de la raza Tabloncillo en $\mathrm{F}_{2}$. La siembra para la caracterización se hizo en el Campo Experimental CUCBA bajo condiciones de temporal, en parcelas de dos surcos con 40 plantas a una densidad de 50 mil plantas por hectárea. 
Se consideraron 36 caracteres; 13 de planta que fueron: inicio de floración masculina (X1), inicio de floración femenina (X2), finalización de floración masculina (X3), finalización de floración femenina (X4), $50 \%$ de floración masculina (X5), $50 \%$ de floración femenina (X6), número de hojas (X7), longitud de la hoja de la mazorca principal (X12), ancho de la hoja de la mazorca principal (X13), altura de planta (X14), altura de mazorca (X15), área de la hoja de la mazorca principal (X31) y área foliar por planta (X32). Siete características de espiga; longitud total (X8), longitud del pedúnculo (X9), longitud de la parte ramificada (X10), número de ramas (X11), longitud de espiguilla (X29), ancho de espiguilla (X30) y longitud de la rama principal (X33); más 16 características de mazorca: número de brácteas de cubierta ("totomoxtle") de la mazorca principal (X16), longitud del pedúnculo de la mazorca principal (X17), longitud de mazorca (X18), diámetro de mazorca (X19), número de hileras de la mazorca (X20), número de granos por hilera (X21), espesor de grano (X22), peso de la mazorca (X23), ancho del grano (X24), longitud del grano (X25), diámetro de olote (X26), peso de grano por mazorca (X27), volumen de grano por mazorca (X28), volumen de grano (X34), porcentaje de olote (X35) y densidad de grano (X36), de acuerdo con Carballo y Benítez (1997).

Con los datos de los 129 materiales y las 36 variables se realizó el análisis de componentes principales con base en Rawlings (1988) y Sánchez (1995), mediante gráficas "biplot". Este tipo de gráficas se han utilizado para simplificar y relacionar en forma conjunta los elementos o grupos de elementos con las variables de estudio (Morales et al., 2007). En esta ocasión los materiales se agruparon inicialmente en cuatro intervalos de altitud de los sitios de colecta, 0 a 1000, 1001 a 1800,1801 a 2200 , y arriba de $2200 \mathrm{~m}$

Para conocer la interrelación de los materiales se hizo análisis de agrupamiento de los 129 maíces con 36 variables, al utilizar como medida de similitud al coeficiente de correlación $r_{i j}=\left(\begin{array}{lll}\Sigma_{k} & x_{k i} & x_{k j}\end{array}\right) /\left(\sum_{k} x_{k i}{ }^{2} \Sigma_{k} x_{k j}{ }^{2}\right)^{1 / 2}$, donde $i, j$ corresponden a las colectas $i$ y $j$, y $k$ a las variables bajo estudio. Los valores $r_{i j}$ se calcularon después de estandarizar las variables $\left(x_{k j}\right)$ con media cero y varianza 1 . Con los valores $r_{i j}$ se hizo el agrupamiento de las accesiones con el método Promedio de Grupo (UPGMA), mediante el paquete estadístico NTSYS versión 2.1.

\section{RESULTADOS Y DISCUSIÓN}

\section{Evaluación}

Hubo diferencias significativas entre colectas en todas las variables analizadas en los tres ambientes de evaluación. En el análisis de varianza combinado hubo diferen- cias significativas entre localidades, variedades e interacción localidad x variedad, en todas las variables, con excepción de mazorcas dañadas y calificación de mazorca, variables que sólo fueron medidas en el CUCBA y en Las Garzas.

En el CUCBA, que fue el mejor ambiente con una media de $3972 \mathrm{~kg} \mathrm{ha}^{-1}$, la entrada más rendidora fue LUG03 x LUG14 (Cuadro 1) con un rendimiento de grano de $6711 \mathrm{~kg} \mathrm{ha}^{-1}$ seguida de M05027 (Tuxpeño) con $6193 \mathrm{~kg} \mathrm{ha}^{-1}$. M05027 proviene de La Ciénega, Guachinango, Jal., donde es conocido como Criollo Tampiqueño, es de grano blanco dentado y se usa principalmente para elaborar tortillas (Ron et al., 2006). M05021 (Тabloncillo) presentó un rendimiento de grano de $5794 \mathrm{~kg}$ ha $^{-1}$ con características agronómicas aceptables, como calidad de mazorca y de los más altos porcentajes de grano. Algunas colectas pertenecientes a la raza Ancho (M05002, M05020 y M05099) introducidas a Jalisco, posiblemente de los Estados de Guerrero y Morelos, tuvieron un rendimiento de grano sobresaliente si se considera su valor especial en el mercado de grano para la preparación de "pozole", un platillo nacional popular.

En Las Garzas, que fue la localidad tropical, se tuvo un rendimiento medio de $2535 \mathrm{~kg} \mathrm{ha}^{-1}$, y los testigos mejorados REMACO-38 (que substituyó a INIFAP48 en esta localidad) y LUG03 x LUG14 fueron los de mayor rendimiento de grano (Cuadro 1); sin embargo, algunas poblaciones nativas tuvieron rendimientos comparables, como M05100 (Criollo Liso) originario de La Hacienda del Cabezón, Ameca, Jal., que se consume localmente en forma de "elote" a pesar de su grano duro semicristalino (Ron et al., 2006).

En Tepatitlán de Morelos, Jal., donde las lluvias fueron escasas al inicio y durante la floración, se tuvo un rendimiento medio de apenas $1207 \mathrm{~kg} \mathrm{ha}^{-1}$; la colecta que rindió más fue M05089 (Elotes Occidentales) con $3166 \mathrm{~kg}$ ha $^{-1}$, originaria de Ahualulco del Mercado, Jal., donde se siembra de temporal en "coamiles" (terrenos de ladera sembrados con coa), cuyo principal uso es para "pozole" y se comercializa principalmente en Guadalajara (Ron et al., 2006). El híbrido testigo mejorado LUG03 x LUG14 fue afectado por la sequía y fue superado en rendimiento de grano por 12 accesiones, generalmente más precoces, así como por la cruza entre las colectas representativas de la raza Tabloncillo (JAL43 x JAL263) en $F_{1}$ y $F_{2}$. Los resultados en esta localidad reflejan la importancia de los maíces criollos, especialmente cuando los factores del ambiente no son favorables para el crecimiento y desarrollo; al mismo tiempo confirman el escaso valor de los maíces mejorados en estas condiciones de cultivo, especialmente por su ciclo más tardío, y resaltan la necesidad 
de obtener maíces precoces mejorados para aprovechar

y Hallauer, 1989).

los efectos de la interacción variedades $\mathrm{x}$ ambientes (Ron

Cuadro 1. Rendimiento por ambiente y a través de los tres ambientes y características agronómicas promedio de los ambientes de evaluación. Zapopan, Las Garzas y Tepatitlán, 2005.

\begin{tabular}{|c|c|c|c|c|c|c|c|c|c|c|c|c|c|c|c|c|c|}
\hline Ent. & Material & \multicolumn{4}{|c|}{ Rendimiento $\left(\mathrm{kg} \mathrm{ha}^{-1}\right)$} & FM & $\mathrm{FF}$ & $\mathrm{AP}^{\ddagger}$ & $\mathrm{AM}$ & $\mathrm{CM}$ & $\mathrm{AR}^{\ddagger}$ & $\mathrm{AT}^{\ddagger}$ & MXP & $\mathrm{MD}^{\ddagger}$ & LM & $\mathrm{DM}$ & G \\
\hline 90 & M05100 (TAP) & 3894 & 5251 & 4281 & 2151 & 66 & 68 & 268 & 144 & 61 & 6 & 35 & 0.9 & 7.5 & 15.8 & 3.8 & 80 \\
\hline 23 & M05021 (TAB) & 3640 & 5794 & 3981 & 1146 & 74 & 78 & 311 & 200 & 63 & 21 & 18 & 0.7 & 9.8 & 14.8 & 3.8 & 85 \\
\hline 42 & M05040 (CEL) & 3376 & 4990 & 2548 & 2588 & 68 & 71 & 291 & 180 & 46 & 21 & 31 & 0.8 & 15.6 & 13.9 & 4.2 & 79 \\
\hline 44 & M05042 (GAV) & 3374 & 6087 & 2041 & 1994 & 69 & 73 & 294 & 184 & 47 & 29 & 24 & 0.6 & 18.5 & 14.3 & 4.4 & 84 \\
\hline 87 & $\mathrm{TAB} . \mathrm{RC}_{1}$ & 3371 & 4825 & 3251 & 2036 & 67 & 69 & 252 & 141 & 56 & 5 & 40 & 1.0 & 11.1 & 14.5 & 3.7 & 80 \\
\hline 62 & INIFAP14 (TAP) & 3307 & 4297 & 3443 & 2181 & 64 & 66 & 246 & 151 & 58 & 24 & 29 & 0.8 & 7.9 & 13.5 & 3.7 & 86 \\
\hline 25 & M05023 (TAB) & 3281 & 4074 & 2872 & 2898 & 64 & 66 & 265 & 151 & 60 & 18 & 36 & 0.8 & 9.4 & 15.2 & 3.3 & 87 \\
\hline 43 & M05041 (CEL) & 3218 & 4691 & 2346 & 2618 & 68 & 70 & 276 & 165 & 48 & 28 & 24 & 0.8 & 17.8 & 13.6 & 4.2 & 82 \\
\hline 71 & INIFAP31 (TUX) & 3216 & 5933 & 3104 & 610 & 80 & 84 & 339 & 217 & 60 & 36 & 18 & 0.7 & 11.1 & 15.1 & 4.1 & 79 \\
\hline 70 & INIFAP30 (TUX) & 3210 & 4943 & 3825 & 861 & 76 & 79 & 311 & 197 & 59 & 13 & 23 & 0.7 & 9.6 & 15.0 & 3.8 & 85 \\
\hline 41 & M05039 (CEL)) & 3209 & 5230 & 1755 & 2643 & 68 & 72 & 295 & 176 & 49 & 24 & 31 & 0.7 & 12.3 & 12.5 & 4.5 & 81 \\
\hline 50 & M05089 (EOC) & 3105 & 4076 & 2073 & 3166 & 65 & 68 & 275 & 161 & 51 & 28 & 36 & 0.8 & 15.1 & 15.7 & 3.6 & 81 \\
\hline 47 & M05045 (CEL) & 3100 & 5744 & 2217 & 1339 & 75 & 79 & 319 & 212 & 58 & 28 & 24 & 0.6 & 11.3 & 14.7 & 4.2 & 81 \\
\hline 28 & M05026 (GAV) & 3092 & 5053 & 3134 & 1088 & 69 & 72 & 293 & 178 & 60 & 20 & 31 & 0.7 & 10.1 & 14.4 & 4.1 & 80 \\
\hline 35 & M05033 (TAB) & 3079 & 4925 & 3428 & 885 & 70 & 73 & 291 & 180 & 58 & 20 & 25 & 0.8 & 9.0 & 14.1 & 3.6 & 82 \\
\hline 15 & M05013 (TAB) & 3072 & 5341 & 2316 & 1558 & 76 & 80 & 324 & 198 & 49 & 26 & 28 & 0.6 & 21.1 & 16.2 & 3.9 & 80 \\
\hline 20 & M05018 (TAB) & 2975 & 5024 & 3316 & 585 & 73 & 77 & 320 & 198 & 58 & 29 & 20 & 0.6 & 10.0 & 14.9 & 3.8 & 82 \\
\hline 38 & M05036 (TAB) & 2922 & 3878 & 3185 & 1703 & 65 & 67 & 275 & 156 & 61 & 6 & 27 & 0.8 & 7.1 & 14.2 & 3.4 & 83 \\
\hline 86 & TAB. Típico $\left(\mathrm{F}_{1}\right)$ & 2895 & 3321 & 2507 & 2858 & 62 & 64 & 250 & 146 & 48 & 39 & 30 & 0.9 & 12.6 & 14.7 & 3.4 & 84 \\
\hline 14 & M05012 (GAV) & 2871 & 4707 & 3267 & 640 & 75 & 78 & 304 & 200 & 66 & 29 & 18 & 0.6 & 7.3 & 14.1 & 4.0 & 77 \\
\hline 66 & INIFAP25 (TAP) & 2871 & 3767 & 3546 & 1299 & 69 & 71 & 287 & 173 & 57 & 14 & 35 & 0.8 & 11.1 & 16.1 & 3.6 & 79 \\
\hline 63 & INIFAP22 (TAB) & 2839 & 4017 & 3668 & 833 & 71 & 74 & 283 & 178 & 55 & 30 & 25 & 0.7 & 11.3 & 14.2 & 4.0 & 81 \\
\hline 16 & M05014 (TUX) & 2825 & 4842 & 3108 & 527 & 79 & 84 & 326 & 209 & 57 & 37 & 15 & 0.5 & 13.6 & 15.1 & 4.3 & 78 \\
\hline 34 & M05032 (GAV) & 2806 & 4292 & 3434 & 693 & 76 & 80 & 309 & 198 & 62 & 21 & 33 & 0.7 & 7.4 & 14.2 & 3.8 & 80 \\
\hline 24 & M05022 (TAB) & 2712 & 4144 & 2088 & 1903 & 64 & 66 & 276 & 161 & 49 & 23 & 37 & 0.7 & 13.9 & 15.6 & 3.5 & 82 \\
\hline 85 & TAB. Típico $\left(\mathrm{F}_{2}\right)$ & 2706 & 3244 & 2265 & 2610 & 61 & 64 & 239 & 137 & 49 & 19 & 36 & 0.9 & 16.8 & 14.5 & 3.3 & 82 \\
\hline 9 & M05006 (TAB) & 2681 & 4186 & 2378 & 1478 & 66 & 69 & 285 & 165 & 60 & 30 & 26 & 0.7 & 18.2 & 15.3 & 4.0 & 79 \\
\hline 59 & INIFAP8 (TAB) & 2666 & 2538 & 3481 & 1979 & 66 & 68 & 277 & 147 & 53 & 18 & 38 & 0.8 & 12.4 & 16.4 & 3.4 & 83 \\
\hline 1 & M04001 (TAB) & 2654 & 3552 & 2395 & 2015 & 64 & 66 & 272 & 158 & 48 & 30 & 25 & 0.7 & 17.2 & 14.8 & 3.5 & 82 \\
\hline 64 & INIFAP23 (TAP) & 2633 & 3868 & 3059 & 970 & 67 & 69 & 271 & 163 & 58 & 8 & 29 & 0.7 & 6.1 & 14.6 & 3.6 & 81 \\
\hline 55 & M05094 (TAP) & 2629 & 2847 & 3234 & 1807 & 61 & 63 & 249 & 129 & 58 & 16 & 35 & 0.8 & 10.3 & 16.0 & 3.4 & 84 \\
\hline 30 & M05028 (TUX) & 2620 & 5228 & 2157 & 475 & 77 & 81 & 339 & 211 & 54 & 23 & 20 & 0.5 & 12.9 & 15.3 & 4.1 & 81 \\
\hline 22 & M05020 (ANC) & 2594 & 5075 & 1775 & 933 & 71 & 75 & 305 & 187 & 47 & 41 & 28 & 0.6 & 20.1 & 14.4 & 4.1 & 77 \\
\hline 89 & M05099 (ANC) & 2589 & 4524 & 1436 & 1808 & 68 & 71 & 297 & 179 & 45 & 34 & 34 & 0.7 & 27.6 & 14.0 & 4.2 & 81 \\
\hline 11 & M05008 (TUX) & 2583 & 4632 & 2727 & 391 & 81 & 86 & 327 & 219 & 52 & 44 & 14 & 0.5 & 15.8 & 14.8 & 5.0 & 78 \\
\hline 51 & M05090 (TAP) & 2579 & 2835 & 3092 & 1810 & 60 & 63 & 241 & 128 & 58 & 10 & 36 & 0.8 & 9.9 & 15.2 & 3.4 & 84 \\
\hline 75 & INIFAP41 (ELS) & 2576 & 3164 & 3287 & 1278 & 67 & 70 & 274 & 148 & 55 & 11 & 37 & 0.8 & 11.2 & 17.9 & 3.4 & 79 \\
\hline 27 & M05025 (TUX) & 2570 & 4553 & 2201 & 956 & 75 & 80 & 315 & 204 & 50 & 34 & 26 & 0.5 & 16.3 & 15.6 & 4.2 & 80 \\
\hline 3 & M04003 (TUX) & 2569 & 4538 & 3060 & 110 & 81 & 86 & 337 & 226 & 59 & 31 & 22 & 0.6 & 10.7 & 15.5 & 3.4 & 75 \\
\hline 67 & INIFAP26 (ONA) & 2538 & 4082 & 3103 & 431 & 73 & 77 & 286 & 165 & 63 & 19 & 28 & 0.6 & 7.3 & 15.3 & 3.8 & 80 \\
\hline 26 & M05024 (TAB) & 2503 & 2783 & 1836 & 2890 & 60 & 63 & 256 & 136 & 47 & 31 & 36 & 0.7 & 19.1 & 14.0 & 3.5 & 86 \\
\hline 13 & M05011 (TAB) & 2496 & 2988 & 3350 & 1149 & 67 & 69 & 265 & 148 & 60 & 29 & 28 & 0.7 & 11.3 & 15.4 & 3.6 & 82 \\
\hline
\end{tabular}




\begin{tabular}{|c|c|c|c|c|c|c|c|c|c|c|c|c|c|c|c|c|c|}
\hline 53 & M05092 (TAP) & 2493 & 2754 & 2578 & 2145 & 59 & 62 & 228 & 119 & 62 & 21 & 28 & 0.9 & 6.6 & 14.9 & 3.0 & 87 \\
\hline 60 & INIFAP11 (TUX) & 2436 & 4457 & 2608 & 243 & 83 & 88 & 333 & 226 & 53 & 24 & 23 & 0.6 & 14.9 & 14.6 & 3.2 & 82 \\
\hline 10 & M05007 (ELS) & 2373 & 4184 & 2345 & 588 & 73 & 78 & 319 & 196 & 59 & 31 & 28 & 0.6 & 11.5 & 14.8 & 3.7 & 80 \\
\hline 74 & INIFAP40 (CEL) & 2314 & 3243 & 2941 & 757 & 69 & 71 & 280 & 163 & 57 & 10 & 37 & 0.7 & 10.1 & 16.9 & 3.4 & 76 \\
\hline 48 & M05053 (EOC) & 2289 & 2900 & 1369 & 2599 & 66 & 69 & 266 & 161 & 52 & 32 & 31 & 0.6 & 23.9 & 15.3 & 3.9 & 80 \\
\hline 6 & M05003 (JAL) & 2249 & 5440 & 858 & 448 & 81 & 88 & 361 & 234 & 42 & 33 & 23 & 0.4 & 25.7 & 16.3 & 4.2 & 77 \\
\hline 83 & INIFAP57 (BOF) & 2223 & 2725 & 3464 & 479 & 78 & 82 & 270 & 174 & 60 & 8 & 27 & 0.8 & 9.8 & 14.2 & 3.3 & 81 \\
\hline 88 & M05098 (ELS) & 2180 & 4038 & 1923 & 579 & 78 & 82 & 294 & 196 & 48 & 28 & 27 & 0.6 & 21.2 & 15.1 & 3.7 & 78 \\
\hline 46 & M05044 (TAB) & 2154 & 4093 & 1321 & 1049 & 71 & 76 & 310 & 195 & 45 & 37 & 25 & 0.6 & 22.4 & 14.9 & 3.9 & 78 \\
\hline 76 & INIFAP42 (GAV) & 2138 & 3173 & 2552 & 689 & 70 & 72 & 286 & 161 & 50 & 7 & 36 & 0.7 & 17.2 & 18.1 & 3.3 & 75 \\
\hline 68 & INIFAP27 (ONA) & 2084 & 2205 & 3208 & 839 & 68 & 70 & 267 & 146 & 58 & 16 & 35 & 0.7 & 10.1 & 16.7 & 3.1 & 77 \\
\hline 57 & INIFAP5 (TAB) & 2082 & 2246 & 2495 & 1505 & 62 & 65 & 257 & 134 & 57 & 11 & 36 & 0.8 & 8.8 & 15.5 & 3.3 & 83 \\
\hline 61 & INIFAP12 (TUX) & 2078 & 3773 & 2298 & 165 & 85 & 89 & 338 & 220 & 55 & 33 & 25 & 0.6 & 13.0 & 15.4 & 3.1 & 78 \\
\hline 40 & M05038 (TUX) & 1796 & 3144 & 1924 & 318 & 82 & 87 & 320 & 218 & 53 & 11 & 25 & 0.6 & 8.7 & 12.6 & 3.2 & 79 \\
\hline 18 & M05016 (PEP) & 1778 & 3093 & 1849 & 391 & 81 & 86 & 332 & 218 & 43 & 43 & 28 & 0.4 & 20.2 & 13.1 & 4.3 & 80 \\
\hline 8 & M05005 (TUX) & 1693 & 3110 & 1732 & 238 & 85 & 90 & 342 & 230 & 56 & 33 & 30 & 0.6 & 10.7 & 14.7 & 3.4 & 82 \\
\hline 81 & INIFAP55 (TUX) & 1664 & 3303 & 1443 & 247 & 84 & 90 & 348 & 235 & 53 & 31 & 22 & 0.5 & 10.9 & 14.4 & 3.1 & 80 \\
\hline 19 & M05017 (ELS) & 1626 & 2992 & 1584 & 303 & 81 & 86 & 314 & 205 & 48 & 15 & 27 & 0.5 & 16.7 & 19.7 & 3.5 & 78 \\
\hline 77 & INIFAP46 (TAB) & 1547 & 2644 & 1713 & 283 & 77 & 81 & 296 & 184 & 57 & 9 & 40 & 0.5 & 15.5 & 14.6 & 3.5 & 77 \\
\hline 84 & INIFAP58 (BOF) & 1231 & 2163 & 880 & 650 & 79 & 84 & 297 & 189 & 47 & 15 & 36 & 0.6 & 14.9 & 14.3 & 3.2 & 73 \\
\hline 39 & M05037 (GAV) & 924 & 1772 & 778 & 223 & 84 & 90 & 324 & 227 & 50 & 29 & 26 & 0.4 & 9.2 & 13.7 & 3.4 & 83 \\
\hline 12 & M05009 (BOF) & 892 & 1801 & 449 & 426 & 83 & 89 & 322 & 215 & 33 & 35 & 24 & 0.4 & 24.3 & 15.8 & 3.5 & 71 \\
\hline 17 & M05015 (BOF) & 634 & 1588 & 228 & 88 & 88 & 94 & 302 & 203 & 27 & 16 & 29 & 0.3 & 22.4 & 16.1 & 3.4 & 72 \\
\hline 49 & M05056 (TAB) & 274 & 548 & 100 & 175 & 82 & 88 & 295 & 208 & 12 & 48 & 20 & 0.1 & 28.7 & 12.5 & 3.9 & 79 \\
\hline
\end{tabular}

Ent. $=$ Número de entrada. ${ }^{+}$Grupo racial entre paréntisis. TAP $=$Tabloncillo Perla; TAB $=$Tabloncillo; TUX $=$Tuxpeño; CEL $=$Celaya; GAV $=$Generación avanzada; $\mathrm{ANC}=$ Ancho; ONA $=$ Onaveño; EOC $=$ Elotes Occidentales; JAL = Jala; ELS = Elotero de Sinaloa; BOF $=$ Bofo; PEP $=$ Pepiti1la. $\stackrel{\ddagger}{*}$ Promedio de Zapopan y Las Garzas. $\mathrm{Z}=$ Zapopan; $\mathrm{G}=$ Las Garzas; $\mathrm{T}=$ Tepatitlan; FM = Floración masculina (días); FF $=$ Floración femenina (días); AP = Altura de planta (cm); AM = Altura de mazorca (cm); CM = Calificación de mazorca, escala de 10 a 100,100 lo mejor; AR = Acame de raíz (\%); AT = Acame de tallo (\%); MXP = Mazorcas por planta; $\mathrm{MD}=$ Mazorcas dañadas (\%); LM = Longitud de mazorca $(\mathrm{cm}) ; \mathrm{DM}=\mathrm{Diámetro} \mathrm{de}$ mazorca $(\mathrm{cm}) ; . \mathrm{G}=$ Porcentaje de grano (\%).

A través de los tres ambientes, el testigo mejorado (LUG03 x LUG14) fue el de mayor rendimiento (4491 kg ha $\left.^{-1}\right)$; cuatro accesiones presentaron rendimientos sobresalientes, que podrían considerarse estables, entre los que destacan M05100 (3894 $\mathrm{kg} \mathrm{ha}^{-1}$ ) por su precocidad (66 y $68 \mathrm{~d}$ a floración masculina y femenina, respectivamente), bajo nivel de acame de raíz (6 \%) y sanidad de mazorca; así como M05021 (3640 kg ha-1) por su elevado porcentaje de grano $(85 \%)$. Las poblaciones de mayor precocidad a floraciones masculina y femenina, fueron: M05092 (59 y 62 d), M05091 (60 y 62 d), M05090 (60 y 63 d), pertenecientes a la raza Tabloncillo Perla, y M05024 (60 y 63 d) de Tabloncillo Amarillo; todas fueron ligeramente más precoces que la cruza entre tabloncillos típicos (JAL43 x JAL263) en $F_{1}$ y $F_{2}$. El maíz más tardío fue la colecta M05015 (Coreño, de la Raza Bofo) que tuvo 88 y 94 d a floraciones masculina y femenina, respectivamente. INIFAP 14, M05023, M05024 y M05092 mostraron los va- lores más altos de porcentaje de grano, 86, 87, 86 y 87 $\%$, respectivamente, y podrían ser una buena fuente para la incorporación de este carácter a materiales mejorados. La colecta con la posición de la mazorca más alta fue M05004 (Jala) con $235 \mathrm{~cm}$, y las de posición más baja fue la colecta M05092 (Maíz Jazmín, de la Raza Tabloncillo con fuerte influencia de Tabloncillo Perla) con $123 \mathrm{~cm}$, y la cruza simple testigo (LUG03 x LUG14) con $118 \mathrm{~cm}$.

Tabloncillo $\mathrm{RC}_{1}$ mostró rendimiento de grano más alto, mayor sanidad de mazorca y menor acame que la cruza entre las colectas típicas de Tabloncillo (JAL43 y JAL263) en $F_{1}$ y $F_{2}$, pero fue más tardío y con menor porcentaje de grano. La cruza entre las dos colectas típicas representativas de la raza Tabloncillo tuvo valores similares en las generaciones $\mathrm{F}_{1}$ y $\mathrm{F}_{2}$; el bajo grado de heterosis es un reflejo de la baja divergencia genética entre estas dos colectas que resulta de la presencia de alelos 
comunes en las dos colectas referidas representativas de la raza Tabloncillo, de las que JAL43 ha sido utilizada en otros estudios (Sánchez et al., 2000).

La colecta de mayor rendimiento de los maíces para usos especiales fue M05002 de grano pozolero de la raza Ancho, con un rendimiento de grano de $3330 \mathrm{~kg} \mathrm{ha}^{-1}$; M0589 perteneciente a la raza Elotes Occidentales de grano morado que se consume en "elote" y también en "pozole", rindió $3105 \mathrm{~kg} \mathrm{ha}^{-1}$. En el caso de los maíces negros que se consumen principalmente en "elote", INIFAP41 (Elotero de Sinaloa) fue el de mayor rendimiento con $2576 \mathrm{~kg} \mathrm{ha}^{-1}$. INIFAP56, INIFAP58, M05009 y M05015 de la raza Bofo de grano con textura harinosa para la preparación de todo tipo de alimentos tradicionales, presentaron rendimientos de grano de 1960, 1231, 892 y $634 \mathrm{~kg} \mathrm{ha}^{-1}$, respectivamente, los más bajos en la evaluación. En general, estos maíces fueron muy tardíos, de portes de planta altos, se acamaron, baja calidad de grano y mazorca y bajo porcentaje de mazorcas por planta.

\section{Caracterización}

Los dos primeros componentes principales explicaron $55 \%$ de la variabilidad total. Según la Figura 1, el grupo de colectas con menor dispersión fue el correspondiente a los criollos colectados en el rango de altitud arriba de $2200 \mathrm{~m}$, que sobresalen principalmente por su mayor espesor del grano (X22) y longitud de la rama principal de la espiga (X33), que son los vectores que se ubican en la parte media del grupo; así como por sus menores valores en longitud de la parte ramificada de la espiga (X10), número de brácteas de cubierta ("totomoxtle") de la mazorca principal (X16), peso de la mazorca (X23), peso del grano de la mazorca (X27) y volumen del grano de la mazorca (X28), que corresponden a los vectores variable más opuestos a este grupo. El grupo más disperso en la Figura 1 fue el de las poblaciones colectadas en el rango de altitud de 0 a $1000 \mathrm{~m}$, seguido del grupo de 1001 a $1800 \mathrm{~m}$; las poblaciones del rango de 1801 a $2200 \mathrm{~m}$ estuvieron prácticamente integradas con el grupo de las colectadas arriba de $2200 \mathrm{~m}$.

Las variables más importantes para definir el primer componente principal, con base en la longitud y posición de los vectores en las primeras dos dimensiones (Figura 1), fueron: área foliar por planta (X32), número de hojas (X7) y número de ramas de la espiga (X11), con valores de $0.9085,0.9073$ y 0.8548 , respectivamente; las más importantes con valores negativos fueron: las longitudes del pedúnculo de la espiga (X9), la rama principal de la espiga (X33), de la espiguilla (X29) y total de la espiga (X8), con valores de $-0.7519,-0.6347,-0.5938$ y
-0.5699, respectivamente. Las variables más importantes en la definición del segundo componente principal fueron, con valores positivos: ancho del grano (X24), volumen del grano por mazorca (X28) y peso de grano por mazorca (X27), con valores de $0.6416,0.6156$ y 0.6118 , respectivamente; con valores negativos destacaron: floración femenina $50 \%$ (X6), floración femenina final (X4), floración masculina final (X3) e inicio de floración femenina (X2), con valores de $-0.6602,-0.6557,-0.6370$ y -0.6325 , respectivamente.

En el dendrograma (Figura 2) se identificaron tres grupos principales con 0.0 de coeficiente de correlación. El Grupo 1 (parte superior) estuvo constituido por 55 materiales que al promediar la altitud de los sitios de colecta, dieron un valor de $953 \mathrm{~m}$; sin embargo el rango de altitud fue de 67 a $2107 \mathrm{~m}$. Este grupo se caracterizó por tener valores altos en: número de hojas (22), número de ramas de la espiga (25), altura de planta $(315 \mathrm{~cm})$, altura de mazorca $(220$ $\mathrm{cm})$, peso de la mazorca (145 g), peso de grano por mazorca $(122 \mathrm{~g})$, el volumen de grano por mazorca $(164 \mathrm{~mL})$ y área foliar por planta $\left(182 \mathrm{~cm}^{2}\right)$; y por sus valores bajos en longitud total de la espiga $(65 \mathrm{~cm})$ y en longitud del pedúnculo de la mazorca $(8.65 \mathrm{~cm})$. Entre las razas representadas en este grupo las de mayor frecuencia fueron Tuxpeño, Celaya, Bofo y Jala, y las generaciones avanzadas de híbridos comerciales.

En este grupo se identificaron dos poblaciones de la raza Tuxpeño con influencia de otras razas, que se han estado sembrando y reconociendo por los productores como tipos más o menos definidos por sus características de mazorca. Uno de estos tipos corresponde a los maíces Chinos (M04002, M04003, M05005, INIFAP11, INIFAP12, INIFAP32 e INIFAP55) colectados principalmente en el municipio de Ruiz, Nay., que se caracterizan por sus mazorcas cilíndricas alargadas de grano blanco (Ron et al., 2006); el otro tipo corresponde a los maíces Tampiqueños (M05008, M05014, M05025, M05027, M05028 e INIFAP31) colectados principalmente en los municipios de Guachinango y San Sebastián del Oeste, cuyas mazorcas son gruesas, alargadas y de grano blanco (Ron et al., 2006). Los maíces chinos y tampiqueños, por su similitud con los maíces de la raza Tuxpeño, podrían considerarse como tipos estables relacionados con Tuxpeño. Tabloncillo $\mathrm{RC}_{1}$ se localizó en este grupo junto a las colectas INIFAP26 (Onaveño) e INIFAP35 (Tabloncillo Perla) de Sinaloa y M05033 (Tabloncillo) de la Costa de Jalisco, muy distante de la cruza entre las colectas típicas representativas de la raza Tabloncillo, que se ubicó en otro grupo (Figura 2). Aquí también se localizaron ocho colectas consideradas como tabloncillos que se relacionaron con otras razas, debido probablemente a la infiltración germoplásmica de tipos diferentes y a la selección de los productores. 


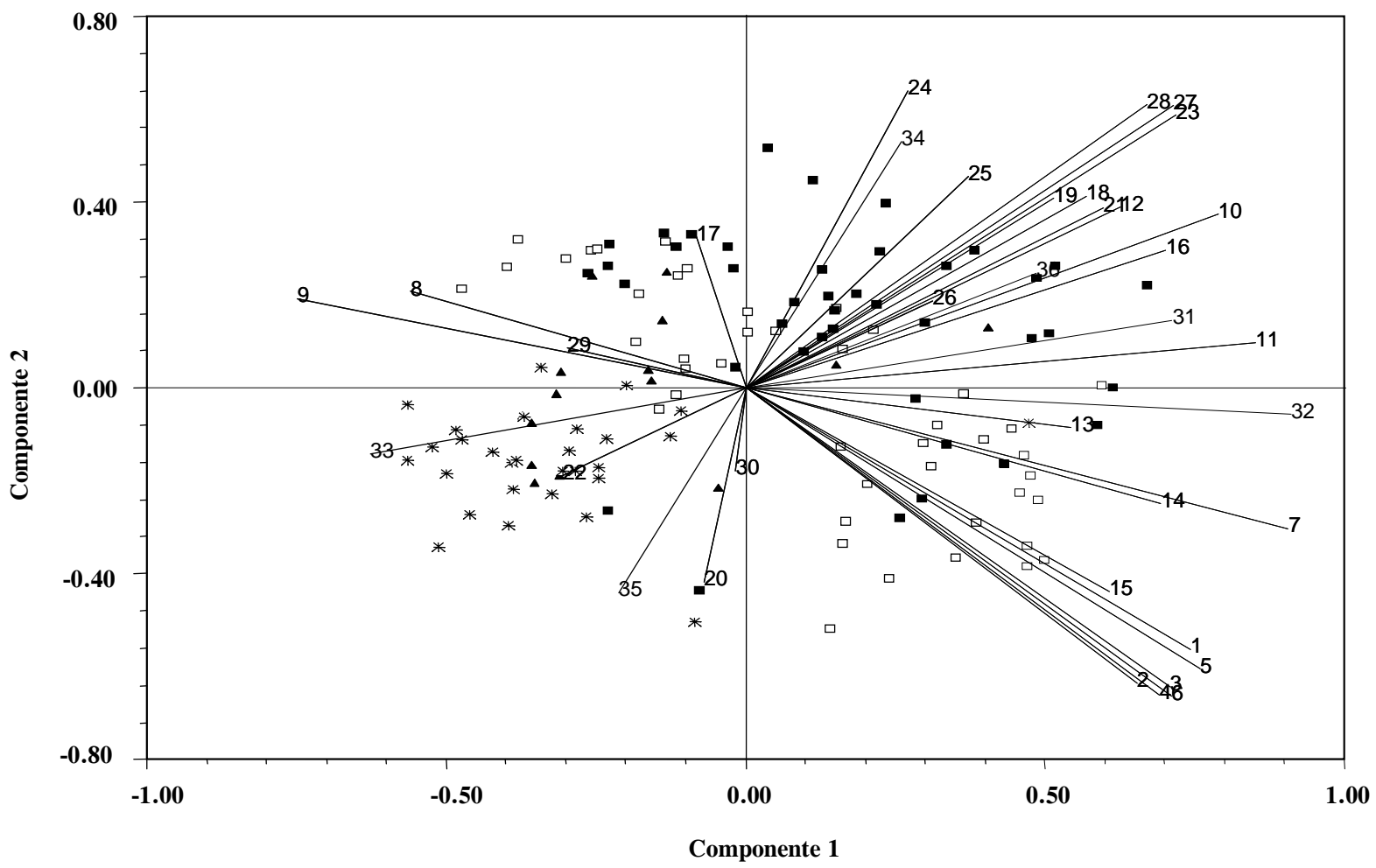

Figura 1. Distribución de 129 materiales sobre los dos primeros componentes principales y 36 variables (líneas contínuas con el número de la variable), por rangos de altitud: 0 a $1000 \mathrm{~m}(\square)$, de 1001 a $1800 \mathrm{~m}(\square)$, de 1801 a $2200 \mathrm{~m}(\Delta)$ y arriba de $\operatorname{los} 2200 \mathrm{~m}(*)$.

El Grupo 2 (parte media del dendrograma) incluyó 33 maíces que provienen de una altitud media de $802 \mathrm{~m}$ y un rango de 77 a $1677 \mathrm{~m}$, que se distinguió por su precocidad (67 y $70 \mathrm{~d}$ a floración masculina y femenina, respectivamente), porte bajo de planta $(262 \mathrm{~cm})$, posición baja de la mazorca principal $(162 \mathrm{~cm})$ y un reducido número de hileras (9). En este grupo predominaron las colectas representativas de Tabloncillo de altitudes intermedias y Tabloncillo Perla de las regiones costeras de los Estados de Nayarit y Sinaloa. La cruza entre las dos colectas típicas de Tabloncillo (TAB. TIPICO) estuvo estrechamente relacionada con M05023 (Tabloncillo) e INIFAP14 (Tabloncillo Perla); la primera, podrían utilizarse como típica representativas de la raza Tabloncillo. Por la cercanía con el TAB. TIPICO, M04001, M05001 y M05034 también podrían ser utilizadas en segunda opción, como representativas de la raza Tabloncillo de grano blanco, al igual que M05006 y M05011 de grano amarillo (Figura 2).

El Grupo 3 (parte inferior del dendrograma) se constituyó por 41 accesiones procedentes de partes altas de Michoacán, casi todas de la raza Mushito, más dos de Maíz Dulce y dos de Elotes Cónicos; esta relación ya había sido reportada por Sánchez et al. (2000) con base en datos morfológicos y de isoenzimas. Este grupo se caracterizó por presentar valores altos en: longitud del pedúnculo de la espiga $(28 \mathrm{~cm})$, longitud de la rama principal de la espiga $(36 \mathrm{~cm})$ y porcentaje de olote $(20 \%)$; y valores bajos en: longitud de la parte ramificada de la espiga $(10 \mathrm{~cm})$, número de ramas de la espiga (12), longitud de la hoja de la mazorca principal $(98 \mathrm{~cm})$, número de hojas de la mazorca principal (8), longitud de la mazorca $(14 \mathrm{~cm})$, número de granos por hilera (25), peso de la mazorca (79 $\mathrm{g})$, peso de grano por mazorca $(64 \mathrm{~g})$, volumen de grano por mazorca $(96 \mathrm{~mL})$ y densidad de grano $\left(0.67 \mathrm{~g} \mathrm{~mL}^{-1}\right)$. Estos valores promedio reflejan problemas de adaptación de los maíces de la raza Mushito a Zapopan, Jal., donde se sembraron para la caracterización.

M05020 y M05099 de la raza Ancho y destinados especialmente al mercado de grano "pozolero", mostraron una relación muy cercana (Figura 2), a pesar de que se colectaron en sitios diferentes; se ubicaron en el Grupo 2 y se relacionaron con M05044 de la raza Tabloncillo destinado también a "pozole". M05002 también de la raza Ancho y destinado especialmente al grano "pozolero" estuvo en el Grupo 1, relacionado con los maíces tardíos destinados a "pozole" de la raza Tabloncillo (M05013 y M05018). Esta raza Ancho ha sido asociado con los maíces de Ocho hileras del Occidente y Noreste de México (Tabloncillo, Tabloncillo Perla, Elotes Occidentales) por Sánchez et al. (2000). Es evidente la infiltración de 
maíces de grano grande de raza Ancho en la región centro occidente de México, como también ha ocurrido en otras regiones del país (Herrera-Cabrera et al., 2004), debido a la demanda de granos anchos en el mercado nacional de grano para "pozole".

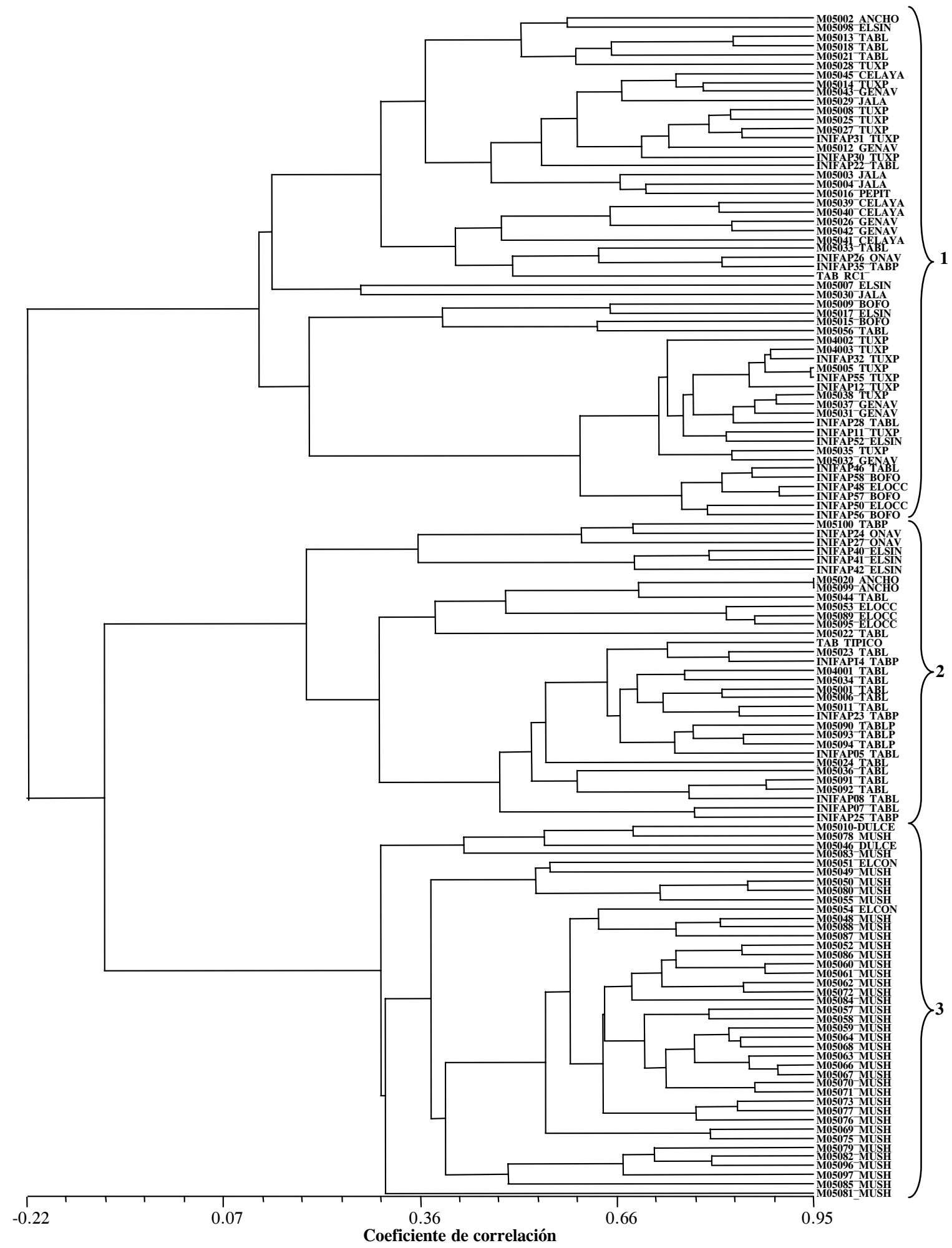

Figura 2. Dendrograma de 129 maíces con 36 variables que forman tres grupos (1, 2 y 3) con 0 de coeficiente de correlación. 
Las características más distintivas de algunas razas, con base en las 36 características evaluadas, fueron: Ancho mostró el grano más ancho $(13 \mathrm{~mm})$, más largo (15 $\mathrm{mm})$, la longitud más corta de la rama principal de la espiga $(24 \mathrm{~cm})$, mayor volumen de grano $(911 \mathrm{~mL})$ y menor porcentaje de olote $(14 \%)$. Bofo fue distintivo por su ciclo tardío a la floración masculina (89 d) y femenina (93 d). Celaya se distinguió por su mayor peso de mazorca $(174 \mathrm{~g})$, peso de grano por mazorca (149 g) y volumen de grano por mazorca $(193 \mathrm{~mL})$. Dulce tuvo pocas ramificaciones de la espiga (11) y menores valores en longitud de mazorca (13 $\mathrm{cm})$, peso de mazorca (67 g), peso de grano por mazorca $(51 \mathrm{~g})$ y volumen de grano por mazorca $(84 \mathrm{~mL})$. Elotes Cónicos sobresalió por menor área foliar por planta (12 888 $\mathrm{cm}^{2}$ ). Jala fue la de mayores valores en altura de planta (362 $\mathrm{cm})$, posición de la mazorca $(261 \mathrm{~cm})$, número brácteas de la mazorca principal (14) y longitud de mazorca $(19 \mathrm{~cm})$. Mushito fue la raza de mayores longitudes de la espiga (74 $\mathrm{cm})$ y del pedúnculo de la espiga $(29 \mathrm{~cm})$, así como menores valores en longitud de la parte ramificada de la espiga $(10 \mathrm{~cm})$, número de ramas de la espiga (12), longitud de la hoja de la mazorca principal $(97 \mathrm{~cm})$, número de brácteas de la mazorca principal (8) y volumen de grano $(403 \mathrm{~mL})$, debido a su falta de adaptación a las condiciones de Zapopan, Jal. Pepitilla presentó el mayor número de hojas al igual que Tuxpeño (24), así como los mayores valores de número de ramas de la espiga (29), longitud de la hoja de la mazorca principal $(114 \mathrm{~cm})$, número de hileras de grano (16), y peso de mazorca (174 g) al igual que Celaya, área de la hoja de la mazorca principal $\left(917 \mathrm{~cm}^{2}\right)$, área foliar por planta $\left(21457 \mathrm{~cm}^{2}\right)$ y porcentaje de olote $(28 \%)$; estos valores se obtuvieron de una sola colecta. Tabloncillo fue el segundo en precocidad (70 y $73 \mathrm{~d}$ a floración masculina y femenina, respectivamente). Tabloncillo Perla fue el más precoz a floración masculina (66 d) y femenina (68 d), y el de menor porte de planta $(253 \mathrm{~cm})$ y posición de la mazorca $(153 \mathrm{~cm})$. Tuxpeño presentó el mayor número de granos por hilera (37) y menor longitud de espiguilla $(8 \mathrm{~cm})$.

\section{CONCLUSIONES}

Se identificaron criollos, como M05100 (Maíz Liso), M05027 (Maíz Tampiqueño) y M05021 (Maíz Tabloncillo Blanco), que presentaron rendimientos de grano comparables al testigo mejorado (LUG03 x LUG14), pero éste fue superado por criollos precoces en la localidad con mayores limitaciones de humedad. Los maíces utilizados para "pozole" y "elote" que tuvieron mayores rendimiento de grano fueron M05002 (Ancho), M05089 (Elotes Occidentales), INIFAP41 (Elotero de Sinaloa) e INIFAP56 (Bofo), pero fueron tardíos, altos en porte de planta, de baja calidad de grano y mazorca y bajo porcentaje de mazorcas por planta, en comparación con los maíces mejora- dos y con los criollos destinados a la producción de grano para tortillas blancas.

La colecta M05023 de grano amarillo podría utilizarse como típica representativa de la raza Tabloncillo, y como una segunda opción las colectas M04001, M05001 y M05034 de grano blanco, y M05006 y M05011 de grano amarillo. La retrocruza de Tabloncillo $\left(\mathrm{RC}_{1}\right)$ perdió las características distintivas de la raza, pero fue más rendidora y con mejores características agronómicas que los maíces pertenecientes a la raza Tabloncillo.

Los maíces Tampiqueños de los municipios de Guachinango y San Sebastián del Oeste, Jal. y los Chinos del Municipio de Ruiz, Nay. se relacionaron a los maíces de la raza Tuxpeño, y los criollos provenientes de generaciones avanzadas de híbridos comerciales con las colectas de la raza Celaya y Tuxpeño, principalmente.

Con base en el análisis de componentes principales, el número de hojas y área foliar por planta fueron las características con mayor valor descriptivo de la variabilidad fenotípica entre los maíces nativos estudiados.

\section{AGRADECIMIENTOS}

Al Sistema Nacional de Recursos Fitogenéticos (SINAREFI) de la Secretaría de Agricultura, Ganadería, Desarrollo Rural, Pesca y Alimentación (SAGARPA) y a Pioneer Hi-Bred Internacional, Inc., por sus apoyos con recursos financieros para la realización de este trabajo.

\section{BIBLIOGRAFÍA}

Carballo C A, A Benítez V (1997) Manual Gráfico para la Descripción Varietal del Maíz (Zea Mays L.) Secretaría de Agricultura, Ganadería, Desarrollo Rural, Pesca y Alimentación. Servicio Nacional de Inspección y Certificación de Semillas. Colegio de Postgraduados. $70 \mathrm{p}$.

Esteva G, C Marielle (2003) Sin Maíz no hay País. Consejo Nacional para la Cultura y las Artes (CONACULTA). México, D.F. $346 \mathrm{p}$.

González A S (2006) Mitos del Maíz. Artes de México. Número 79. 80 p.

Herrera Cabrera B E, F Castillo González, J J Sánchez González, J M Hernández Casillas, R A Ortega Pazka, M M Goodman (2004) Diversidad del maíz Chalqueño. Agrociencia 38: 191206.

Morales R M M, J Ron P, J J Sánchez G, J L Ramírez D, L De La Cruz L, S Mena M, S A Hurtado De La P y M Chuela B (2007) Relaciones fenotípicas y heterosis entre híbridos comerciales y germoplasma exótico de maíz en Jalisco, México. Rev. Fitotec. Mex 30:285-294.

Muñoz O A (2003) Centli Maíz. Colegio de Posgraduados. Montecillo, Edo. de México. 211 p.

Rawlings J O (1988) Applied Regression Analysis. Wadswoth \& Brooks/Cole Advanced Books \& Software. Pacific Grove, California. $553 \mathrm{p}$. 
Ron P J, A R Hallauer (1989) Interacciones ambientales de 23 variedades de maíz en la región de El Bajio, México. Agric. Téc. Méx. 15:3-22.

Ron P J, J J Sánchez G, A A Jiménez C, J A Carrera V, J G Martín L, M M Morales R, L De La Cruz L, S A Hurtado De La P, S Mena M, J G Rodríguez F (2006) Maíces Nativos del Occidente de México. I Colectas 2004. Scientia-CUCBA 8:1139.
Sánchez G J J (1995) El análisis Biplot en clasificación. Rev. Fitotec. Mex. 18:188-203.

Sánchez G J J, M M Goodman, C W Stuber (2000) Isozymatic and morphological diversity in the races of maize of México. Econ. Bot. 54:43-59. 